\title{
Exact Crossing Minimization
}

\author{
Christoph Buchheim ${ }^{1}$, Dietmar Ebner ${ }^{2}$, Michael Jünger ${ }^{1}$, Gunnar W. Klau ${ }^{3}$, \\ Petra Mutzel ${ }^{4}$, and René Weiskircher ${ }^{5}$ \\ 1 Department of Computer Science, University of Cologne \\ \{buchheim, mjuenger\}@informatik.uni-koeln.de \\ 2 Institute of Computer Graphics and Algorithms, Vienna University of Technology \\ ebner@ads.tuwien.ac . at \\ 3 Department of Mathematics and Computer Science, FU Berlin \\ gunnar@math.fu-berlin.de \\ 4 Department of Computer Science, University of Dortmund \\ petra.mutzel@uni-dortmund.de \\ 5 CSIRO Mathematical and Information Sciences \\ Rene.Weiskircher@csiro.au
}

\begin{abstract}
The crossing number of a graph is the minimum number of edge crossings in any drawing of the graph into the plane. This very basic property has been studied extensively in the literature from a theoretic point of view and many bounds exist for a variety of graph classes. In this paper, we present the first algorithm able to compute the crossing number of general sparse graphs of moderate size and present computational results on a popular benchmark set of graphs. The approach uses a new integer linear programming formulation of the problem combined with strong heuristics and problem reduction techniques. This enables us to compute the crossing number for 91 percent of all graphs on up to 40 nodes in the benchmark set within a time limit of five minutes per graph.
\end{abstract}

\section{Introduction}

Crossing minimization is among the oldest and most fundamental problems arising in the areas of automatic graph drawing and VLSI design. At the same time, it is very easy to formulate: "Given a graph $G=(V, E)$, draw it in the plane with a minimum number of edge crossings". A drawing of $G$ is a mapping of each vertex $v \in V$ to a distinct point and each edge $e=(v, w) \in E$ to a curve connecting the incident vertices $v$ and $w$ without passing through any other vertex. Common points of two edges that are not incident vertices are called crossings. The minimum number of crossings among all drawings of $G$ is denoted by $\operatorname{cr}(G)$.

The main goal in automatic graph drawing is to obtain a layout that is easy to read and understand. Although the definition of layout quality often depends on the particular application and is hard to measure, the number of edge crossings is among the most important criteria [18. Figure 1 shows a comparison of different drawings for the same graph preferring different aesthetic criteria.

In fact, the crossing minimization problem is even older than the area of automatic graph drawing. It goes back to P. Turán, who proposed the problem 


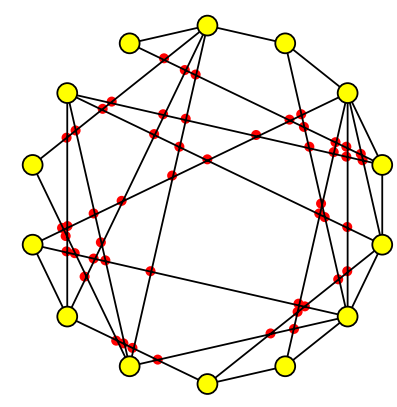

(a)

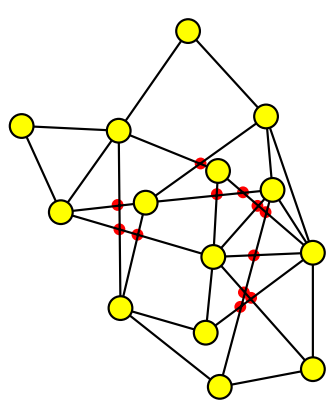

(b)

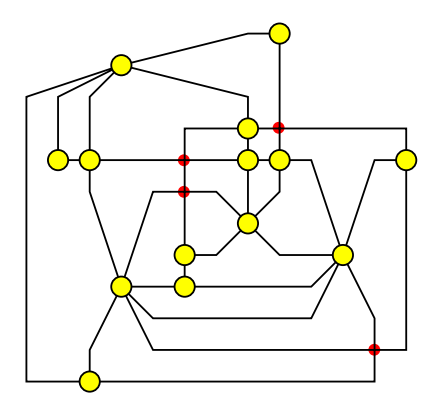

(c)

Fig. 1. Three drawings of the same graph with 51 (a), twelve (b), and four crossings (c). Most aesthetic criteria like few edge bends, uniform edge lengths, or a small drawing area favor the first two drawings, while the last drawing is preferable with respect to the number of edge crossings.

in his "Notes of Welcome" in the first issue of the Journal of Graph Theory [19]. While working in a labor camp during the Second World War, he noted that crossings of the rails between kilns and storage yards caused the trucks to jump the rails. Minimizing these crossings corresponds to the crossing minimization problem for a complete bipartite graph $K_{m, n}$.

In 1953, K. Zarankiewicz and K. Urbaník independently claimed a solution for this problem by providing a drawing rule for complete bipartite graphs $K_{m, n}$ with $\left\lfloor\frac{m}{2}\right\rfloor\left\lfloor\frac{m-1}{2}\right\rfloor\left\lfloor\frac{n}{2}\right\rfloor\left\lfloor\frac{n-1}{2}\right\rfloor$ crossings. About ten years later, their proof of optimality was shown to be wrong and it is still unknown whether the conjecture holds. The situation for complete graphs $K_{n}$ is similar. Their crossing number is conjectured to be $\frac{1}{4}\left\lfloor\frac{n}{2}\right\rfloor\left\lfloor\frac{n-1}{2}\right\rfloor\left\lfloor\frac{n-2}{2}\right\rfloor\left\lfloor\frac{n-3}{2}\right\rfloor$, which has been verified for graphs of up to ten nodes by Guy [10. However, both conjectures are based on a drawing rule and therefore serve as an upper bound for $\operatorname{cr}(G)$.

It is well known that the general crossing minimization problem is NP-hard 7 . More precisely, it is shown that the crossing number problem, i.e., "given a graph $G$ and $a$ non-negative integer $K$, decide whether there is a drawing of $G$ with at most $K$ edge crossings", is NP-complete. However, for fixed $K$, we can obtain a polynomial time algorithm by examining all possible configurations with up to $K$ crossings. Clearly, this algorithm is not appropriate in practical applications for larger values of $K$. Recently, Grohe could show that this problem can be solved in time $O\left(|V|^{2}\right)$ [8]. Even though the exponent is independent of $K$, the constant factor of his algorithm grows doubly exponentially in $K$. Therefore, this method is also of little relevance in practice.

The search for approximation algorithms did not lead to significant results either. While there is no known polynomial time approximation algorithm with any type of quality guarantee for the general problem, Bhatt and Leighton could derive an algorithm for graphs with bounded degree that approximates the number of crossings plus the number of nodes in polynomial time [2. Due to the 
complexity of the crossing minimization problem, many restricted versions have been considered in the literature. However, in most cases, e.g., for bipartite, linear, and circular drawings, the problem remains $N P$-hard [6, 16, 15].

The most prominent and practically successful approach for solving the crossing minimization problem heuristically is the planarization approach [1, which addresses the problem by a two step strategy. The idea is to remove a preferably small number of edges in order to obtain a planar subgraph and reinsert them into a planar drawing with as few crossings as possible. For each step, various algorithms can be applied. Pre- and post-processing procedures have been developed to improve the solution quality. A computational study on state-of-the-art heuristics can be found in [9].

Contribution and Structure. In this paper, we present the first algorithm able to compute the crossing number of general sparse graphs of moderate size. We state computational results on a popular benchmark set of graphs, the so-called Rome library [5]. The approach uses a new integer linear programming formulation of the problem combined with strong heuristics and problem reduction techniques. This enables us to compute the crossing number for 91 percent of all graphs on up to 40 nodes in the Rome library within a time limit of five minutes per graph. In Sect. 2, we show how to reduce the problem to the easier problem of computing crossing-minimal drawings where each edge is involved in at most one crossing. We give an integer linear programming formulation for the simpler problem and a branch-and-cut algorithm to compute provably optimal solutions for this formulation in Sect. 3. Section 4 summarizes the computational results obtained with our new approach for the simple as well as the general crossing number problem for Rome library graphs. We present conclusions and further work in Section 5 .

\section{Reduction to Simple Drawings}

The area of crossing minimization is closely related to the field of planarity testing, which aims to decide whether a given graph $G$ can be drawn in the plane without any edge crossings. This task can be performed surprisingly fast, more precisely in linear time [11,4]. Beyond doubt, one of the ground-breaking results in this research area was Kuratowski's theorem, which provides a full characterization of planar graphs based on the complete graph $K_{5}$ and the complete bipartite graph $K_{3,3}$.

Theorem 1 (Kuratowski's theorem). A finite graph is planar if and only if it contains no subgraph that is a subdivision of $K_{5}$ or $K_{3,3}$.

We can obtain a subdivision $S$ of a graph $G$ by repeatedly replacing its edges by a path of length two.

As a consequence of Theorem 1] at least two edges in every Kuratowski subdivision, i.e., a subdivision of $K_{5}$ or $K_{3,3}$, have to cross in every planar drawing of a graph $G$. As we describe in Section 3 , we can obtain inequalities from this 
observation that fully characterize the set of realizable crossing configurations (corresponding to drawings in the plane).

Unfortunately, even deciding whether there is a drawing for a given set of edge crossings is NP-complete [13]. This problem is known as the realizability problem and can be stated as follows: "Given a set of edge pairs D, does there exist a drawing of $G$ such that two edges $e, f \in E$ cross each other if and only if $\{e, f\} \in D$ ?

In order to efficiently answer this question, we also need to know the order of the edge crossings for a particular edge $e$. With this additional information, it is easy to solve the problem by placing dummy vertices on all chosen crossings and testing the resulting graph for planarity.

One way to work around the realizability problem is the reduction to simple drawings. A drawing is called simple if each edge crosses at most one other edge. Not surprisingly, there are graphs that do not admit any simple drawing. Pach and Tóth [17] showed the following more general theorem:

Theorem 2. Let $G=(V, E)$ be a simple graph drawn in the plane so that every edge is crossed by at most $k$ others. If $0 \leq k \leq 4$, then we have

$$
|E| \leq(k+3)(|V|-2)
$$

They could further prove that this bound cannot be improved for $0 \leq k \leq 2$ and that for any $k \geq 1$ the following inequality holds:

$$
|E| \leq \sqrt{16.875 k}|V| \approx 4.108 \sqrt{k}|V|
$$

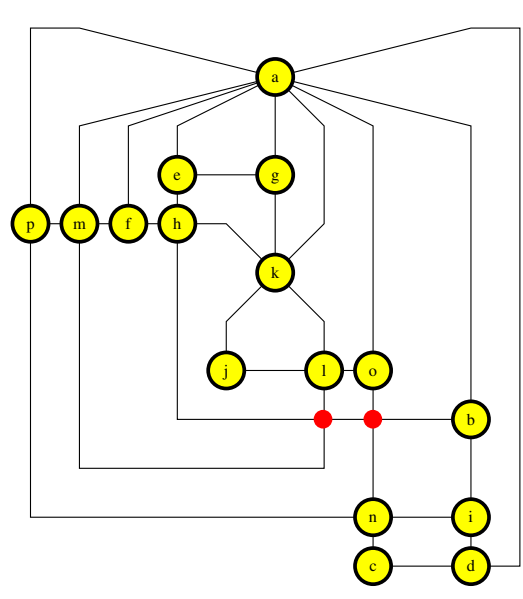

(a)

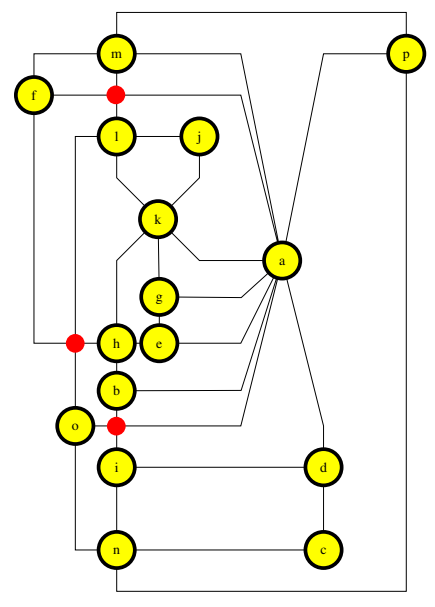

(b)

Fig. 2. Optimal drawing of a graph with two crossings (a) and an optimum simple drawing of the same graph with three crossings (b). Both drawings were produced with our exact algorithm presented in this paper. 
Furthermore, Bodlaender and Grigoriev proved that it is NP-complete to determine whether there is a simple drawing for a given graph $G$ [3. If there is such a drawing, we denote the minimum number of crossings among all simple drawings of $G$ by $\operatorname{crs}(G)$.

Even if there is a simple drawing for $G$, its crossing number $\operatorname{crs}(G)$ does not necessarily coincide with $\operatorname{cr}(G)$. Consider the sample graph in Figure 2, The left drawing shows an optimum drawing with two crossings while the right drawing shows an optimum drawing among all simple drawings.

However, given a graph $G=(V, E)$ we can create a graph $G^{*}=\left(V^{*}, E^{*}\right)$ by replacing every edge $e \in E$ with a path of length $|E|$. It is easy to show that for any non-negative number $K$ the graph $G$ can be drawn with $K$ crossings if and only if there is a simple drawing of $G^{*}$ with $K$ crossings. Therefore, it is "sufficient" to solve the crossing minimization problem restricted to simple drawings in order to solve the general crossing minimization problem, clearly at significant computational expense. Since the transformation obviously can be done in polynomial time, the NP-completeness of the corresponding decision problem for simple drawings follows immediately from the NP-completeness for the general crossing number problem [7].

It is well-known that every graph $G$ admits a good drawing with a minimum number of crossings, i.e., a drawing that satisfies the following conditions:

1. no edge crosses itself

2. adjacent edges do not cross each other

3. non-adjacent edges cross each other at most once

Therefore it is sufficient to replace every edge $e=(v, w) \in E$ with a path of length $|E|-|\delta(v)|-|\delta(w)|-1$. We can further lower the number of required dummy edges by using any upper bound for $\operatorname{cr}(G)$, since no edge can cross more than $\operatorname{cr}(G)$ other edges in any optimal solution.

\section{An Integer Linear Program for Simple Drawings}

Mathematical programming is a powerful tool to address NP-hard combinatorial optimization problems. Starting from an integer linear program (ILP) modeling the problem under consideration, i.e., a linear program with integer variables, sophisticated techniques like branch-and-cut can be applied. In the following, we present an integer linear programming formulation for the crossing minimization problem restricted to simple drawings. It is described in Sect. 2 how this method can be used to solve the general crossing minimization problem.

Let $G=(V, E)$ be a graph and let $D$ be a set of unordered pairs of edges of $G$. We call $D$ simple if for every $e \in E$ there is at most one $f \in E$ such that $(e, f) \in D$. Furthermore, $D$ is called realizable if there is a drawing of $G$ such that there is a crossing between edges $e$ and $f$ if and only if $(e, f) \in D$.

For every graph $G$ and every simple $D$, we denote with $G_{D}$ the graph that is obtained by introducing a dummy node $d_{e, f}$ for each pair of edges $(e, f) \in D$. More precisely, we introduce dummy nodes on both $e$ and $f$ and identify them. 
Note that $G_{D}$ is only well-defined if $D$ is simple, as otherwise it would not be clear where to place the dummy nodes. For both edges $e_{1}$ and $e_{2}$ resulting from splitting $e$, we set $\hat{e}_{1}=\hat{e}_{2}=e$, analogously for $f$.

Corollary 1. Let $D$ be simple. Then $D$ is realizable if and only if $G_{D}$ is planar.

Using a linear time planarity testing and embedding algorithm, we can thus test in time $O(|V|+|D|)$ whether $D$ is realizable, and compute a realizing drawing in the affirmative case.

Definition 1. For a set of pairs of edges $D \subseteq E^{2}$ we define

$$
x_{e, f}^{D}= \begin{cases}1 & \text { if }(e, f) \in D \\ 0 & \text { otherwise } .\end{cases}
$$

Next, for every subgraph $H=\left(V^{\prime}, E^{\prime}\right)$ of $G_{D}$, let $\hat{H}=\left\{\hat{e} \mid e \in E^{\prime}\right\} \subseteq E$. Less formally, $\hat{H}$ contains all edges of $G$ involved in the subgraph $H$ of $G_{D}$.

Proposition 1. Let $D$ be simple and realizable. For an arbitrary simple set of pairs of edges $D^{\prime} \subseteq E^{2}$ of $G=(V, E)$ and any subdivision $H$ of $K_{5}$ or $K_{3,3}$ in $G_{D^{\prime}}$, the following inequality holds:

$$
C_{D^{\prime}, H}: \sum_{(e, f) \in \hat{H}^{2} \backslash D^{\prime}} x_{e f}^{D} \geq 1-\sum_{(e, f) \in \hat{H}^{2} \cap D^{\prime}}\left(1-x_{e f}^{D}\right)
$$

Proof. Suppose (3) is violated. Since $x_{e, f}^{D} \in\{0,1\}$ for all $e, f \in E$, the left hand side of (3) must be zero and the right hand side must be one, which means that

$$
\begin{array}{ll}
x_{e, f}^{D}=0 & \text { for all }(e, f) \in \hat{H}^{2} \backslash D^{\prime}, \text { and } \\
x_{e, f}^{D}=1 & \text { for all }(e, f) \in \hat{H}^{2} \cap D^{\prime} .
\end{array}
$$

It follows from the definition of $x^{D}$ that $\hat{H}^{2} \cap D^{\prime}=\hat{H}^{2} \cap D$, in other words, that $G_{D}$ corresponds to $G_{D^{\prime}}$ on the subgraph induced by $\hat{H}$, so that $H$ is also a forbidden subgraph in $G_{D}$, i.e., a subdivision of $K_{5}$ or $K_{3,3}$. It follows from Kuratowski's Theorem that $G_{D}$ is not planar. This contradicts the realizability of $D$ by Corollary 1 .

Theorem 3. Let $G=(V, E)$ be a simple graph. A set of pairs of edges $D \subseteq E^{2}$ is simple and realizable if and only if the following conditions hold:

$$
\begin{array}{rr}
x_{e, f}^{D} \in\{0,1\} & \forall e, f \in E, e \neq f \\
\sum_{f \in E} x_{e, f}^{D} \leq 1 & \forall e \in E \\
C_{D^{\prime}, H} & \text { for every simple } D^{\prime} \subseteq E^{2} \text { and every } \\
\text { forbidden subgraph } \text { in }_{D_{D^{\prime}}}
\end{array}
$$


Proof. It is easy to see that the constraints from the second row are satisfied if and only if $D$ is simple. It remains to show that a simple $D$ is realizable if and only if the conditions $C_{D^{\prime}, H}$ from the last row hold. For a realizable $D$ every $C_{D^{\prime}, H}$ is satisfied according to Proposition 1 .

We have to show that any non-realizable set $D$ violates at least one of the constraints $C_{D^{\prime}, H}$. It follows from Corollary 1 that $G_{D}$ is not planar if $D$ is not realizable and we know from Theorem 1 that there exists a subdivision $H$ of $K_{5}$ or $K_{3,3}$ in $G_{D}$. Let $D^{\prime}=D$ and consider the constraint $C_{D, H}$ :

$$
C_{D, H}: \sum_{(e, f) \in \hat{H}^{2} \backslash D} x_{e f}^{D} \geq 1-\sum_{(e, f) \in \hat{H}^{2} \cap D}\left(1-x_{e f}^{D}\right)
$$

It follows from the definition of $x^{D}$ that every $x_{e, f}^{D} \in \hat{H}^{2} \backslash D$ is zero, hence the left hand side of (4) is also zero. Since $\hat{H}^{2} \cap D \subseteq D$ we also know that

$$
\sum_{(e, f) \in \hat{H}^{2} \cap D}\left(1-x_{e f}^{D}\right)=0
$$

so that the right hand side of $C_{D, H}$ is one. Thus $C_{D, H}$ is violated.

For every simple and realizable set $D \subseteq E^{2}$, we can compute a corresponding drawing in polynomial time. Thus we can reformulate the crossing minimization problem for simple drawings as "Given a graph $G=(V, E)$, find a simple and realizable subset $D \subseteq E^{2}$ of minimum cardinality". This immediately leads to the following ILP-formulation, where we use $x(F)$ as an abbreviation for the term $\sum_{(e, f) \in F} x_{e, f}$ :

$$
\begin{aligned}
& \min x\left(E^{2}\right) \\
& \text { s.t. } \sum_{f \in E} x_{e, f} \leq 1 \\
& x\left(\hat{H}^{2} \backslash D^{\prime}\right)-x\left(\hat{H}^{2} \cap D^{\prime}\right) \geq 1-\left|\hat{H}^{2} \cap D^{\prime}\right| \quad \text { for every simple } D^{\prime} \\
& \text { and every forbidden subgraph } H \text { in } G_{D^{\prime}} \\
& x_{e, f} \in\{0,1\} \\
& \forall e, f \in E
\end{aligned}
$$

It is clearly impractical to generate all constraints $C_{D, H}$ in advance and solve the ILP in a single step. Instead, we embed the given formulation into a branchand-cut framework, separating violated inequalities dynamically during runtime according to the proof of Theorem 3 .

A crucial factor in this approach is the separation problem: "Given a class of valid inequalities and a vector $y \in \mathbb{R}^{n}$, either prove that $y$ satisfies all inequalities in the class, or find an inequality which is violated by $y . "$. Although we can easily separate violated inequalities for integral solution vectors according to the proof of Theorem 3. the problem is more complex within the branch-and-cut framework since we have to deal with fractional values. A heuristic for separating the inequalities is to round variables to either zero or one, and then check 
for violated inequalities. The problem is that the inequalities produced by this heuristic might not be violated by the current fractional solution. In this case we select a branching variable and split the current problem into two subproblems by setting the branching variable to zero, respectively one. The same is done if no inequalities at all are produced by the separation heuristic.

In some cases, we can omit variables from our ILP. For instance, we can split the graph into its blocks (two-connected components) and solve these blocks independently - the crossing number of a graph is equal to the sum of the crossing numbers of its blocks. Furthermore, it is easy to show that adjacent edges do not cross in an optimal drawing and no edge crosses itself, i.e., we can restrict ourselves to good drawings.

\section{Computational Results}

We have implemented the presented algorithm in $\mathrm{C}^{++}$using the class library $L E D A$ and solve the linear programs arising during the optimization process with the commercial optimization library CPLEX (version 8.1). We have integrated our new algorithm into AGD, a powerful library of Algorithms for Graph Drawing. This enables us to use any of the existing planar layout algorithms to produce a drawing for $G$ with $\operatorname{cr}(G)$ crossings.

In order to decrease the computational expense of many input graphs, we applied a number of correctness preserving pre-processing procedures. These remove edges temporarily that do not influence the crossing number of the resulting graph. More precisely we repeatedly remove nodes of degree one and merge paths such that each vertex except the start and the end vertex has degree two to a single edge. The latter approach cannot be applied if we intend to determine $\operatorname{crs}(G)$, since we may exclude the optimal solutions from consideration.

In order to obtain good upper bounds, we apply additionally to known bounds based on the number of vertices and edges the well known planarization approach to the input graph [1]. Furthermore, we make use of an exact algorithm proposed by Jünger and Mutzel [12] that computes the skewness $\operatorname{sk}(G)$ of $G$. It is defined as the minimum number of edges that must be removed from $G$ in order to obtain a planar subgraph. It is well-known that the crossing number of a graph cannot be smaller than its skewness. Hence we have that $\operatorname{cr}(G) \geq \operatorname{sk}(G)$. Computing the skewness is equivalent to the maximum planar subgraph problem, which was shown to be NP-hard by Liu and Geldmacher [14. However, medium sized instances can be solved to optimality in reasonable computation time.

To test the performance of our new algorithm and to compare its solution quality to heuristic approaches, we used a benchmark set of graphs of the University of Rome III, introduced in [5]. The set contains 11, 389 graphs that consist of 10 to 100 vertices and 9 to 158 edges. These graphs were generated from a core set of 112 "real life" graphs used in database design and software engineering applications. Most of the graphs are sparse, which is a common property in most application areas of automatic graph drawing. The average ratio between the number of edges and the number of nodes of the graphs from the benchmark set is about 1.35 . 
Due to the complexity of the crossing minimization problem we only consider graphs of up to 40 nodes. We need to round the current fractional solution to integer values in order to separate violated inequalities. Therefore we experimented with different strategies and compared their performance against each other.

- $R 1$ We round every value that is greater than $1-\varepsilon$ to one. All other variables are mapped to zero. In our implementation, we used $\varepsilon=10^{-10}$.

- R05 Every variable with a value greater or equal than 0.5 is rounded to one.

- R0208 If the value of a variable is less than 0.2 or greater than 0.8 it is mapped to zero or one, respectively. In the interval $[0.2,0.8]$ a coin flip decides if we round to zero or one.

It turns out that $R 1$ performs best on average and is therefore presented in the following figures. While this strategy often leads to constraints that are already satisfied by the current fractional solution, the generated cuts are usually stronger.

Figure 3 shows the percentage of graphs that could be solved within a time limit of five minutes on an Intel Pentium 4 with $2.4 \mathrm{GHz}$ and $1 \mathrm{~GB}$ of main memory. As expected, the difference between our implementation for $\operatorname{cr}(G)$ (crossing number) and $\operatorname{crs}(G)$ (simple crossing number) grows with the size of the graphs. The smaller number of variables needed for the computation of $\operatorname{crs}(G)$ (where we do not need edge decomposition) leads to a significantly higher number of instances that could be solved within the time limit. While the percentage of

Percentage of solved Instances

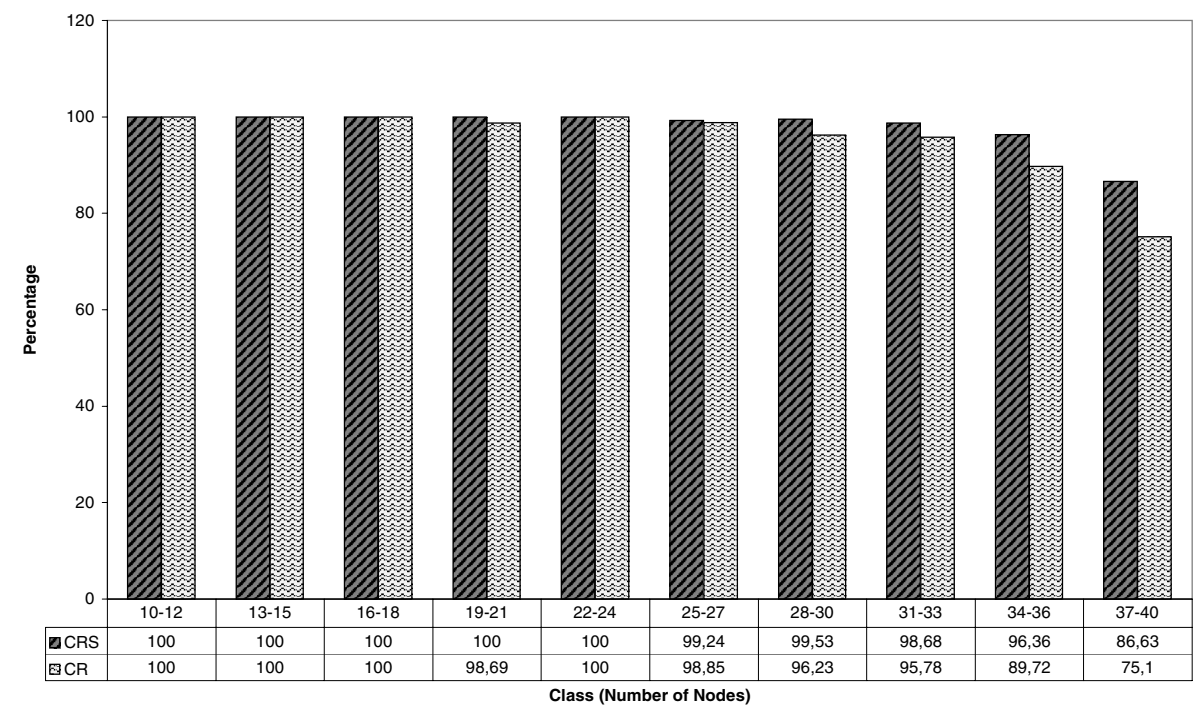

Fig. 3. Percentage of graphs solved by our exact algorithm for graphs on up to 40 nodes with $(C R)$ and without $(C R S)$ supporting multiple crossings per edge 
Table 1. The computation time strongly depends on the number of crossings. The values $K, \bar{t}_{\mathrm{crs}}$, and $\bar{t}_{\mathrm{cr}}$ denote the number of crossings and the average computation time to compute the (simple) crossing-minimal drawings.

\begin{tabular}{crr}
$K$ & $\bar{t}_{\mathrm{cr}}$ & $\bar{t}_{\mathrm{crs}}$ \\
\hline 1 & $0.16 \mathrm{~s}$ & $0.16 \mathrm{~s}$ \\
2 & $6.40 \mathrm{~s}$ & $0.79 \mathrm{~s}$ \\
3 & $52.90 \mathrm{~s}$ & $8.20 \mathrm{~s}$ \\
4 & $155.05 \mathrm{~s}$ & $31.57 \mathrm{~s}$ \\
\hline
\end{tabular}

graphs with 40 nodes where we can compute $\operatorname{cr}(G)$ within the time limit goes down to about $65 \%$, we can still compute $\operatorname{crs}(G)$ for about $80 \%$ of these instances.

Table 1 shows the average computation time for instances that could be solved within five minutes by all of the considered rounding strategies. The required time to solve a particular instance strongly depends on its crossing number, as the table illustrates.

Clearly we are interested in the quality of our results in comparison to heuristic approaches. For the computation of heuristic values we used the planarization approach. Gutwenger and Mutzel presented an extensive computational study of crossing minimization heuristics [9]. The authors investigate the effects of various methods for the computation of a maximal planar subgraph and different edge re-insertion strategies for the planarization approach. Furthermore, they study the impact of post-processing heuristics.

Average Improvement in Comparison to heuristic Results

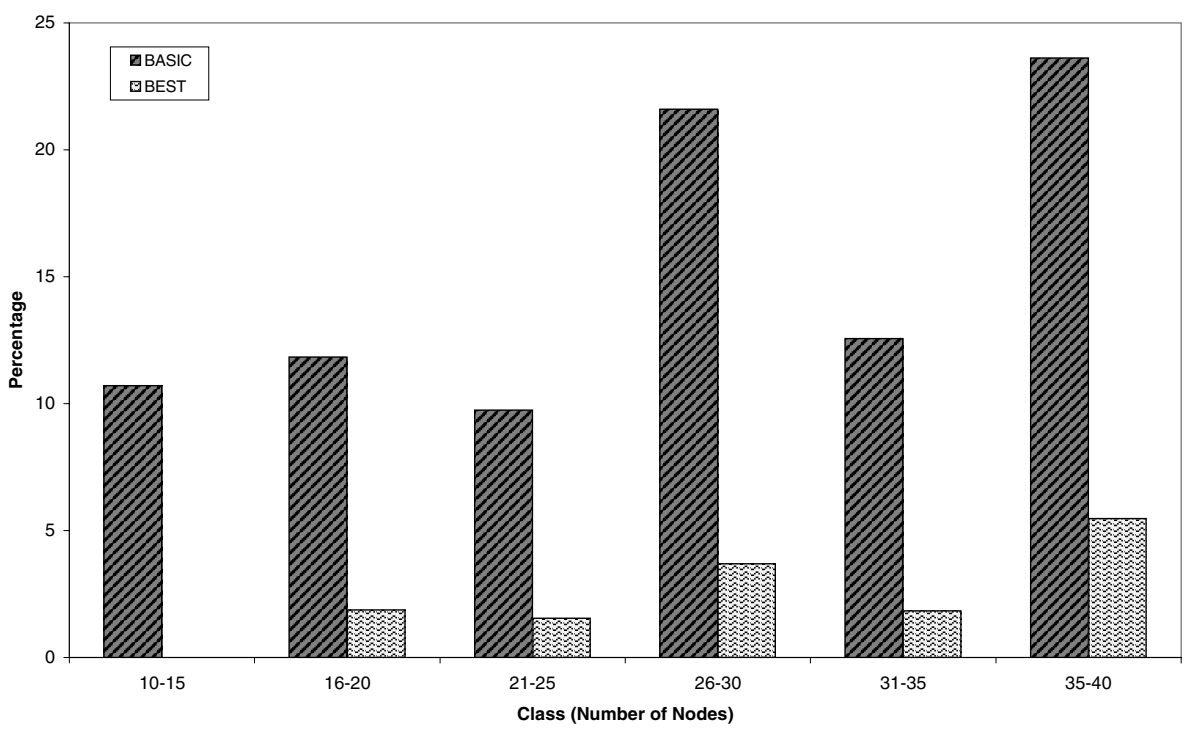

Fig. 4. Comparison between heuristic results and the crossing numbers computed with our exact algorithm 
Figure 4 shows the average improvement in percent that could be achieved in comparison to the basic planarization approach $(B A S I C)$ and the improved version $(B E S T)$, for different classes of graphs. To highlight the improvements, we only considered graphs that could be solved to optimality within the time limit. We can clearly improve the heuristic results for the basic approach, even for the relatively small instances considered in our computational study. Even compared to the best known heuristic methods we achieve a notable improvement for some larger instances. The average improvement over the whole considered benchmark set is about $19.6 \%$ for the basic heuristic and $4.1 \%$ for the best known strategy.

\section{Conclusion and Future Work}

In this paper, we have presented the first algorithm that is able to compute the crossing number for sparse graphs of moderate size. We achieved this by combining a new integer linear programming formulation for the problem with sophisticated problem reduction techniques and the best known heuristics for the problem. Our implementation of the algorithm is able to compute the crossing number for 91 percent of all graphs on up to 40 vertices in a popular benchmark set of graphs within five minutes.

One way of improving the performance of the approach would be to use column generation for subdividing edges. As our computational results have shown, the problem is much easier to solve for simple drawings than for nonsimple drawings. If we found a way of testing efficiently if subdividing a certain edge, and thus allowing more crossings on it, would decrease the overall number of crossings, we could expect to be able to solve much larger problem instances.

Another way forward is studying the polyhedron defined by the set of realizable crossing vectors. By adding new constraints that exclude fractional solutions that are not excluded by our current constraints we should be able to compute the crossing number for larger graphs with more crossings.

\section{References}

1. C. Batini, M. Talamo, and R. Tamassia. Computer aided layout of entityrelationship diagrams. Journal of Systems and Software, 4:163-173, 1984.

2. S. N. Bhatt and F. T. Leighton. A framework for solving VLSI graph layout problems. Journal of Computer and System Sciences, 28:300-343, 1984.

3. H. Bodlaender and A. Grigoriev. Algorithms for graphs embeddable with few crossings per edge. Research Memoranda 036, Maastricht : METEOR, Maastricht Research School of Economics of Technology and Organization, 2004.

4. K. S. Booth and G. S. Lueker. Testing for the consecutive ones property, interval graphs, and graph planarity using PQ-tree algorithms. Journal of Computer and System Sciences, 13(3):335-379, 1976.

5. G. Di Battista, A. Garg, G. Liotta, R. Tamassia, E. Tassinari, and F. Vargiu. An experimental comparison of four graph drawing algorithms. Computational Geometry: Theory and Applications, 7(5-6):303-325, 1997. 
6. P. Eades and N. C. Wormald. Edge crossings in drawings of bipartite graphs. Algorithmica, 11(4):379-403, 1994.

7. M. R. Garey and D. S. Johnson. Crossing number is NP-complete. SIAM Journal on Algebraic and Discrete Methods, 4:312-316, 1983.

8. M. Grohe. Computing crossing numbers in quadratic time. In STOC 2001: Proceedings of the 33rd Annual ACM Symposium on Theory of Computing, 2001.

9. C. Gutwenger and P. Mutzel. An experimental study of crossing minimization heuristics. In G. Liotta, editor, GD 2003: Proceedings of the 11th International Symposium on Graph Drawing, volume 2912 of LNCS, pages 13-24. SpringerVerlag, 2004.

10. R. K. Guy. Crossing numbers of graphs. In Graph Theory and Applications (Proceedings), Lecture Notes in Mathematics, pages 111-124. Springer-Verlag, 1972.

11. J. Hopcroft and R. Tarjan. Efficient planarity testing. Journal of the ACM, 21(4):549-568, 1974.

12. M. Jünger and P. Mutzel. Maximum planar subgraphs and nice embeddings: Practical layout tools. Algorithmica, 16(1):33-59, 1996.

13. J. Kratochvíl. String graphs. II.: Recognizing string graphs is NP-hard. Journal of Combinatorial Theory, Series B, 52(1):67-78, 1991.

14. P. Liu and R. Geldmacher. On the deletion of nonplanar edges of a graph. In Proceedings of the 10th Southeastern Conference on Combinatorics, Graph Theory, and Computing, pages 727-738, Boca Raton, FL, 1977.

15. S. Masuda, T. Kashiwabara, K. Nakajima, and T. Fujisawa. On the NPcompleteness of a computer network layout problem. In Proceedings of the IEEE International Symposium on Circuits and Systems, pages 292-295, 1987.

16. S. Masuda, K. Nakajima, T. Kashiwabara, and T. Fujisawa. Crossing minimization in linear embeddings of graphs. IEEE Transactions on Computers, 39(1):124-127, 1990.

17. J. Pach and G. Tóth. Graphs drawn with few crossings per edge. Combinatorica, 17(3):427-439, 1997.

18. H.C. Purchase. Which aesthetic has the greatest effect on human understanding? In Giuseppe Di Battista, editor, GD '97: Proceedings of the 5th International Symposium on Graph Drawing, volume 1353 of LNCS, pages 248-261. Springer-Verlag, 1997.

19. P. Turán. A note of welcome. Journal of Graph Theory, 1:7-9, 1977. 